\title{
Muscle-Eye-Brain Disease
}

\author{
Anant M. Shenoy, MD*, Jennifer A. Markowitz, MD ${ }^{\star}$, Carsten G. Bonnemann, MD ${ }^{\dagger}$, Kalpathy \\ Krishnamoorthy, MD $¥$, Aaron D. Bossler, MD, PhD§, and Brian S. Tseng, MD, PhD $\ddagger$ \\ * Department of Neurology, Massachusetts General Hospital, Boston, MA \\ † Division of Neurology, The Children's Hospital of Philadelphia, Philadelphia, PA \\ ‡ Division of Child Neurology, Department of Neurology, Massachusetts General Hospital, \\ Boston, MA \\ $\S$ Department of Pathology, Carver College of Medicine, University of lowa, lowa City, lowa
}

\section{Abstract}

A term female infant was evaluated for global developmental delay, hypotonia, hyporeflexia, diffuse weakness including facial muscles, and visual impairment with optic nerve hypoplasia. In the absence of family history or perinatal concerns, an extensive investigation was performed, including lab studies, muscle biopsy, brain MRI and focused genetic testing. This revealed elevated serum CK, a structurally abnormal brain, and a dystrophic-appearing muscle biopsy with evidence of a glycosylation defect in the alpha-dystroglycan complex. Of the 6 known related genes, testing of the POMGnT1 gene showed three heterozygous missense mutations. Thus her history, examination, biopsy specimen, imaging, laboratory, and genetic studies are all consistent with the diagnosis of Muscle-Eye-Brain (MEB) disease. MEB is one of an emerging spectrum of congenital disorders that involve both central and peripheral nervous systems, described further in this case report.

\section{Keywords}

dystroglycanopathy; POMT1; POMT2; POMGnT1; Fukutin; FKRP; LARGE; Walker Warburg; syndrome; Muscle-Eye-Brain disease; fukuyama muscular dystrophy; muscle biopsy; congenital brain malformations; congenital muscular dystrophy

\section{CASE REPORT}

\begin{abstract}
A 6-month-old girl with unremarkable birth history and family history presented with global developmental delay and hypotonia, with inability to lift her head or roll over. At age 8 months, she could not transfer objects between hands and did not babble. Her neurologic examination demonstrated diffuse hypotonia, weakness (including facial muscles), and hyporeflexia. She had poor visual tracking, and ophthalmologic examination revealed optic nerve hypoplasia. Her serum creatine kinase was elevated, ranging from 3000 to $6000 \mathrm{IU} / \mathrm{L}$. A quadriceps muscle biopsy showed marked variation in fiber size, mild increase in endomysial connective tissue, and moth-eaten fibers. Muscle immunohistochemistry revealed normal uniform membranous pattern of staining for dystrophin, merosin, adhalin, beta-sarcoglycan, delta-sarcoglycan, and beta-dystroglycan. A small population of myocytes
\end{abstract}

Reprints: Brian S. Tseng, MD, PhD, Division of Child Neurology, Department of Neurology, Massachusetts General Hospital, 55 Fruit Street, ACC 708, Boston, MA 02114 (bstseng@partners.org).

The authors report no conflicts of interest. 
showed either reduced, absent, or patchy staining for gamma-sarcoglycan. Staining for glycosylated alpha-dystrogylycan, using a glycoepitope-specific antibody, showed reduced immunoreactivity. Hypoglycosylation of alpha-dystroglycan was confirmed by Western blotting of the muscle (Fig. 1). Brain magnetic resonance imaging scan showed diffuse symmetric cortical malformation that was characterized by a nodular-appearing or "cobblestone" cortex (Fig. 2A). There was also abnormal myelination with diffuse T2 hyperintensities involving the deep periventricular and some subcortical white matter (Fig. 2A). The mesencephalon, pons, cerebellum, and medulla were hypoplastic (Fig. 2B). Multiple cerebellar cysts were seen as well (Fig. 2C).

At the age of 20 months, she had two generalized seizures. An electroencephalogram was normal. With her history, she was suspected to have a disorder in the spectrum of Fukuyama congenital muscular dystrophy (CMD) or muscle-eye-brain (MEB) disease. Genetic testing of the POMGnT1 gene showed three heterozygous missense mutations: (1) a 794G>A variation at exon 9 causing an arginine to histidine substitution at codon 265 ; (2) a 932G>A variation at exon 10 causing an arginine to glutamine substitution at codon 311 ; (3) a $1814 \mathrm{G}>\mathrm{A}$ variation at exon 21 causing an arginine to histidine substitution at codon 605 . The c.794G $>$ A and c.932G>A have been shown to be on the same allele in other affected patients. ${ }^{1}$ Normal DNA nucleotide sequence was found for her POMT1, POMT2, FKRP, and FKTN genes. Her history, examination, and diagnostic studies were consistent with the diagnosis of MEB disease.

\section{COMMENT}

This case illuminates the complexity yet greater molecular understanding of dystroglycanopathies, an emerging area of unique genetic disorders that are manifested with involvement of both central (brain and eye) and peripheral nervous system (skeletal muscle). The dystroglycanopathies are a group of muscular dystrophies thought to be caused by reduced glycosylation of alpha-dystroglycan. ${ }^{2,3,5-7}$ Alpha-dystroglycan is a $156-\mathrm{kDa}$ peripheral transmembrane protein that undergoes multiple glycosylation steps, enabling its interaction with extracellular matrix proteins such as laminin, neurexin, and perlecan. ${ }^{2,4}$ Of interest, there does not appear to be a consistent correlation of clinical severity based on the amount of hypoglycosylation on the alpha-dystroglycan. ${ }^{8}$ So far, mutations in six genes (POMT1, POMT2, POMGnT1, Fukutin, FKRP, and LARGE) have been associated with dystroglycanopathy. ${ }^{1-3,5-7}$ These genes with loss-of-function mutations and likely more mutated genes will be identified that can cause a wide range of phenotypic expression ranging from severe congenital onset variants with structural brain malformations (WalkerWarburg Syndrome, MEB, Fukuyama CMD, MDC1D, and overlap phenotypes) to congenital variant with only cerebellar or no structural brain involvement with or without mental retardation (CMD type $1 \mathrm{C}$ and others). In addition, mutations in these genes can lead to a childhood or adult-onset limb-girdle muscular dystrophy variants (LGMD2I, LGMD2L, and LGMD2N). ${ }^{9}$ Classifications of these clinical syndromes are now being annotated with unique clinical, neuroimaging findings and specific loss-of-function gene mutations, some of which can exhibit genetic and phenotypic heterogeneity.

Despite this heterogeneity, these conditions are characterized by generally unique features within a broad spectrum. ${ }^{7}$ Cases of Walker-Warburg Syndrome tend to be seen with severe lissencephaly, hydrocephalus, cerebellar and corpus callosum involvement, severe eye involvement, and typically absent motor development. ${ }^{7}$ As depicted in this case report, MEB/Fukuyama CMD is found usually with preferential frontaparietal pachygyria, polymicrogyria, cerebellar hypoplasia/dysplasia, flattening of the pons, and brainstem plus eye abnormalities. ${ }^{7}$ CMD can be seen with or without cerebellar abnormalities such as cysts or hypoplasia/dysplasia with or without mental retardation, and in a CMD subtype of 
merosin-deficiency, MCD1D, there can be diffuse white matter signal abnormality on brain magnetic resonance imaging scan without mental retardation. ${ }^{7}$

\section{References}

1. Vervoort VS, Holden KR, Ukadike KC, et al. POMGnT1 gene alterations in a family with neurological abnormalities. Ann Neurol 2004;56:143-148. [PubMed: 15236414]

2. Muntoni F, Torelli S, Brockington M. Muscular dystrophies due to glycosylation defects. Neurotherapeutics 2008;5:627-632. [PubMed: 19019316]

3. Muntoni F, Brockington M, Godfrey C, et al. Muscular dystrophies due to defective glycosylation of dystroglycan. Acta Myol 2007;26:129-135. [PubMed: 18646561]

4. Ibraghimov-Beskrovnaya O, Ervasti JM, Leveille CJ, et al. Primary structure of dystrophinassociated glycoproteins linking dystrophin to the extracellular matrix. Nature 1992;355:696-702. [PubMed: 1741056]

5. Peat RA, Smith JM, Compton AG, et al. Diagnosis and etiology of congenital muscular dystrophy. Neurology 2008;71:312-321. [PubMed: 18160674]

6. Mercuri E, Messina S, Bruno C, et al. Congenital muscular dystrophies with defective glycosylation of dystroglycan. A population study. Neurology 2009;72:1802-1809. [PubMed: 19299310]

7. Godfrey C, Clement E, Mein R, et al. Refining genotype-phenotype correlations in muscular dystrophies with defective glycosylation of dystroglycan. Brain 2007;130:2725-2735. [PubMed: 17878207]

8. Jimenez-Mallebrera A, Torelli S, Feng L, et al. A comparative study of alpha-dystroglycan glycosylation in dystroglycanopathies suggests that the hypoglycosylation of alpha-dystroglycan dies not consistently correleate with clinical severity. Brain Pathol 2009;19:596-611. [PubMed: 18691338]

9. Moore SA, Shilling CJ, Westra S, et al. Limb-girdle muscular dystrophy in the United States. J Neuropathol Exp Neurol 2006;65:995-1003. [PubMed: 17021404] 
A

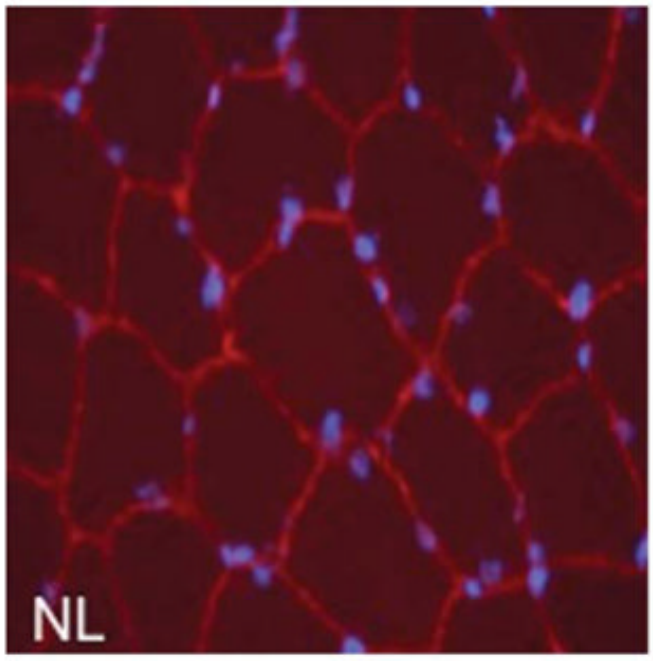

FIGURE 1. $156 \mathrm{kDa})$.
B

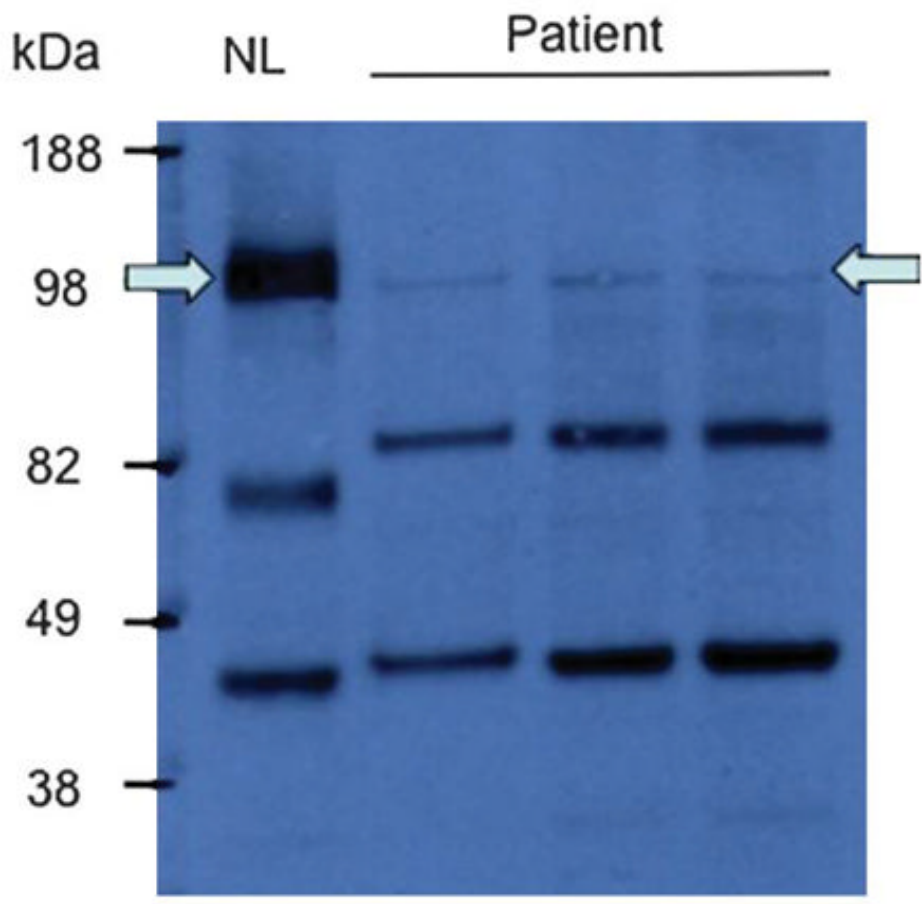

GAPDH

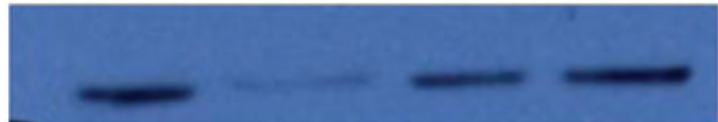

A, Quadriceps biopsy (magnification, 40x) with staining for alpha-dystroglycan for normal control compared with our patient. B, Western blot testing showed decreased abundance of glycosylated alpha-dystroglycan compared with control (arrow) (expected molecular weight 

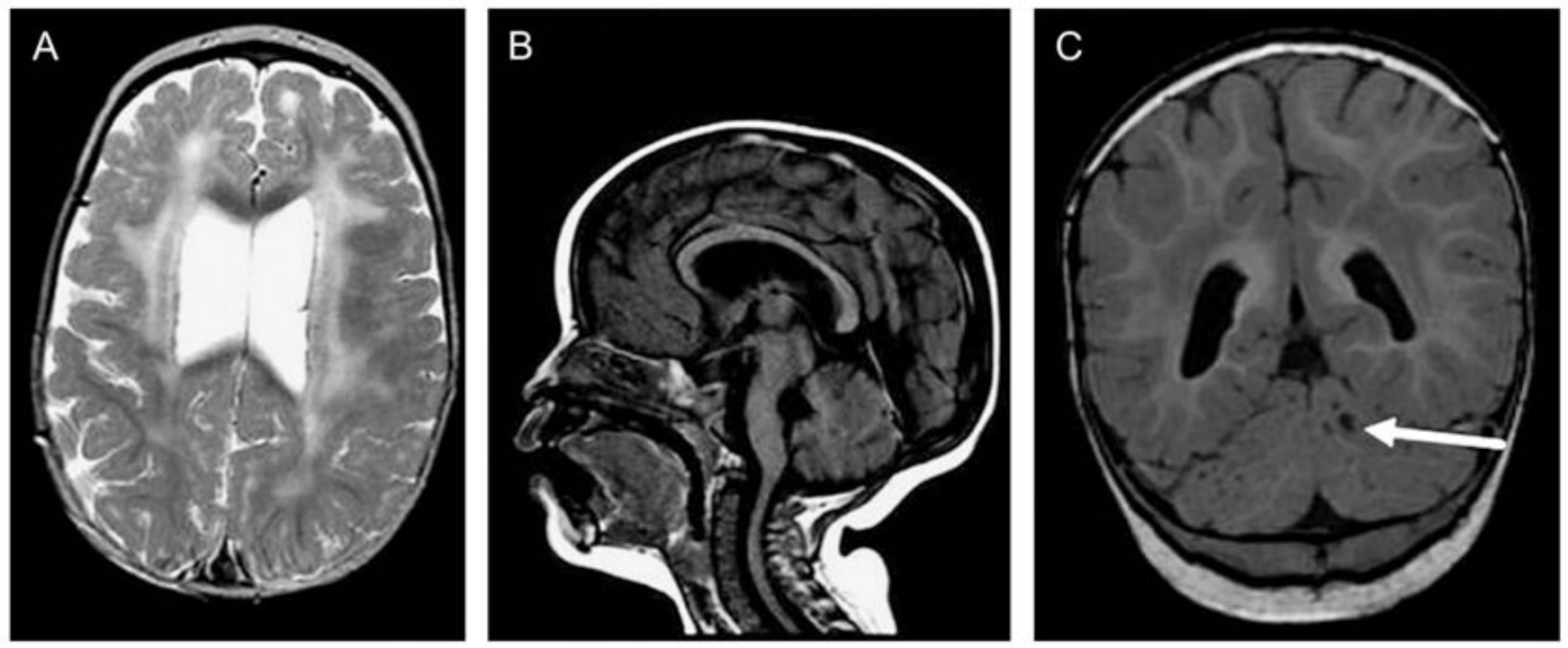

FIGURE 2.

Magnetic resonance imaging of patient's brain. A, Axial T2 image showing diffuse

symmetric cortical malformation characterized by nodular appearing cortex or "cobblestone cortex." There is also abnormal myelination with diffuse hyperintensities involving deep periventricular and subcortical white matter. B, Sagittal T1 image showing hypoplastic mesencephalon, pons, cerebellum, and medulla. Tectum is thickened. C, Coronal FLAIR image showing multiple cerebellar cysts (arrow). 